\title{
FarmaJusta: Dados Abertos Como Suporte à Saúde Pública
}

\author{
Ana Clara Correa ${ }^{1}$, Gustavo Garcia dos Reis Nunes ${ }^{1}$, \\ Larissa da S. Paiva ${ }^{1}$, Renato Cerceau ${ }^{2}$, Sérgio Manuel Serra da Cruz ${ }^{1}$ \\ ${ }^{1}$ Departamento de Computação - Programa de Educação Tutorial PET-SI \\ Universidade Federal Rural do Rio de Janeiro (UFRRJ) - Seropédica, RJ - Brasil \\ ${ }^{2}$ Instituto Alberto Luiz Coimbra de Pós-Graduação e Pesquisa de Engenharia (COPPE) \\ Universidade Federal do Rio de Janeiro (UFRJ) - Rio de Janeiro, RJ - Brasil. \\ \{anaclara, gustavogarcia, larissa, serra\}@pet-si.ufrrj.br, cerceau@ufrj.br
}

\begin{abstract}
Resumo. Diante da percepção da ineficiência da disseminação dos dados abertos relacionados aos preços máximos de medicamentos consumidos pela população brasileira e sobretudo aos eventos de superfaturamento nos preços dos medicamentos no país, percebe-se que há oportunidade de oferecer ferramentas computacionais capazes de auxiliar a disseminação da informação para todos os brasileiros. Para tal, técnicas de computação distribuídas, dados abertos e Interação Humano-Computador foram adotadas no desenvolvimento do FarmaJusta. Este trabalho alia atividades de ensino-pesquisa-extensão e apresenta a ferramenta, bem como analisa os acessos a ferramenta por todos os usuários anônimos.
\end{abstract}

\begin{abstract}
Today, the inefficiency in the dissemination of the open data related to the prices of medicines by the Brazilian government to the population is a critical social issue. It is especially true if we consider the constant occurrence of the ever increasing price of medicines in the country. Thus, to assist Brazilian citizens to find the maximum allowed price of the medicines, we developed a web application that uses the distributed computing, open data and HumanComputer Interaction techniques to aid them. This paper presents our work presents FarmaJusta, discusses of the adoption of these techniques, and analyses the access and usage of the application considering a large set of anonymous users.
\end{abstract}

\section{Introdução}

De acordo com o sexto artigo da Constituição brasileira de 1988 [Brasil 1988], a Saúde é estabelecida como um dos direitos fundamentais da população. O conceito de saúde evoluiu e, atualmente, é definida como o completo bem estar social, físico e mental de um indivíduo. Contudo, de acordo com a Organização das Nações Unidas [Hogerzeil HV 2011], o acesso à medicamentos ainda segue definido como um dos cinco indicadores de garantia ao direito à saúde.

Frequentemente verificam-se episódios de superfaturamento no preço de medicamentos no Brasil. Por exemplo, na cidade de São Paulo, farmácias e drogarias locais aumentaram em até $300 \%$ o preço de determinados produtos [Hebert 2018], afetando a saúde da população local. No intuito de garantir à população brasileira o direito de acesso 
à saúde, existe um órgão responsável por realizar atividades de regulamentação e monitoramento do comércio de medicamentos, ele é conhecido como Câmara de Regulação do Mercado de Medicamentos (CMED) e faz parte da Agência Nacional de Vigilância Sanitária (ANVISA).

Periodicamente a CMED disponibiliza uma lista aberta ao público, ela contém informações sobre o valor máximo que os medicamento podem assumir nas farmácias e drogarias de todo o país. A lista ainda dispõe de informações relacionadas ao Imposto sobre Circulação de Mercadorias e Serviços (ICMS), que podem variar de acordo com o estado no qual o produto é comercializado; princípio ativo do produto; empresa responsável pela fabricação e outras informações.

Todavia, é necessário destacar dois aspectos pertinentes aos inúmeros problemas vivenciados pela sociedade brasileira: o crescimento do acesso dos brasileiros à internet é desigual no campo e nas cidades e há pouca eficiência da democratização do acesso aos dados abertos. Ainda que haja relativa facilidade dos brasileiros acessarem a rede mundial de computadores, muitos não estão cientes da existência das informações e dados abertos disponibilizadas pela CMED no site da ANVISA. Outrossim, a falta de empregabilidade de conceitos de básicos de IHC (Interação Humano Computador) neste site, tais como a usabilidade, acessibilidade e comunicabilidade nos arquivos disponibilizados pela CMED dificulta o entendimento e esclarecimento do usuário diante dos conteúdos exibidos.

Diante desses observações, projetamos e desenvolvemos o FarmaJusta, uma aplicação distribuída e web responsiva (com tamanho adaptado automáticamente de acordo com as dimensões do dispositivo utilizado pelos usuários) que tem por objetivo facilitar a disseminação das ações da CMED para a população brasileira. A ferramenta foi idealizada a fim de atender à parte da população brasileira que possui dificuldades na manipulação de computadores e dispositivos móveis. A aplicação possivelmente facilitará a pesquisa do preço máximo que um medicamento pode assumir legalmente no comércio. O software também disponibiliza um setor administrativo, onde o administrador do serviço terá acesso a análises estatísticas sobre as pesquisas realizadas pelos usuários. Poderá acompanhar o total de acessos e a quantidade de acessos por cada estado; e principalmente poderá consultar quais são os medicamentos mais buscados em nível estadual e nacional.

\section{Trabalhos relacionados}

Atualmente, existem poucos sistemas disponíveis relacionados aos dados abertos na área da saúde pública. Alguns desses software possuem objetivos similares ao do FarmaJusta, que tem como sua principal funcionalidade a responsabilidade de difundir informações relacionadas à medicamentos. Uma dessas aplicações é o ProDoctor Medicamentos, um aplicativo gratuito disponível na Play Store [GooglePlay 2018], que permite o usuário realizar consultas de bulas e de outras informações relacionadas à todos os medicamentos legalizados e aprovados pela ANVISA no Brasil.

Um outro exemplo de aplicativo relacionado à saúde é o Bulário Digital - Medicamentos [GooglePlay 2017], que assim como o ProDoctor, também está disponível para download nas lojas de aplicativos. Ele disponibiliza para pesquisa, informações de mais de 30 mil medicamentos registrados pela ANVISA, e sua principal função é exibir as bulas desses medicamentos. 
Os dois softwares citados, apresentam algumas similares ao FarmaJusta, como por exemplo a facilidade de uso. No entanto, embora que eles auxiliem a propagação de conhecimento no âmbito da saúde pública para a população brasileira, o FarmaJusta se destaca por ser uma plataforma que trata além de disponibilizar de uma maneira mais fácil informações relacionadas ao preço máximo que os medicamentos podem assumir, como também consegue registrar a proveniência das consultas de quais medicamentos são mais pesquisados tanto no cenário estadual como nacional[da Cruz et al. 2009].

Neste sentido, a contribuição do FarmaJusta no presente trabalho é dar suporte gratuito a propagação de informações relacionadas à saúde pública e aos direitos do cidadão brasileiro, com o objetivo de diminuir os eventos no país de superfaturamento no preço de medicamentos, além de também servir como um uma ferramenta de análise dos medicamentos mais pesquisado pela população brasileira.

\section{Metodologia}

Este trabalho adotou a metodologia denominada Fábrica de Software baseada em Métodos Ágeis (FSMA) [Cruz et al. 2014], ela foi concebida para facilitar o desenvolvimento de softwares por grupos que trabalham com atividades extensionistas, tais como o programa PET. Os dados abertos brutos utilizados no sistema são atualizados anualmente pela CMED, e estão estruturados em um arquivo do tipo planilha eletrônica compatível ao programa Excel $®$ 98. A planilha, que possui mais de 54 mil registros, é estruturada em uma tabela contendo os seguintes campos: princípio ativo, composto responsável pela ação, ou seja, pelo efeito farmacológico e está presente em alimentos, plantas e medicamentos; cadastro Nacional da Pessoa Jurídica (CNPJ); nome do laboratório responsável pela fabricação do medicamento; código EAN (Número de Artigo Internacional); descrição do medicamento e entre outros campos.

Para realizar a carga periódica dos dados brutos para a banco, foi necessário realizar uma "limpeza e homogeneização"afim de garantir a correção e integridade do banco de dados. A linguagem de programação Python [FOUNDATION 2018a] ${ }^{1}$ foi protagonista nessa etapa, utilizaram-se três bibliotecas, são as [FOUNDATION 2018b]: 'xlrd', 're' e 'MySQLdb'. ' A biblioteca 're' foi a responsável por fornecer funções para a interpretação de expressões regulares, utilizadas no tratamento de dados.

A biblioteca 'xlrd' proporciona funções manipuláveis de extração de dados de uma planilha em retorno aos parâmetros de nome, folha, colunas e linhas. O Python não tem uma sintaxe literal para expressões regulares, como existe em outras linguagens, logo a biblioteca 're' foi utilizada para fornecer as funções para a interpretação de expressões regulares, sendo útil em momentos onde precisávamos cobrir uma série de termos, expressando apenas o padrão em que elas eram apresentadas. Por exemplo, em um dos casos, criamos uma expressão que diz que apenas os números de um termo deveriam ser extraídos para eliminar os pontos, barras e traços encontrados na coluna CNPJ, que agora passaria a ser tratado como numeral e não mais como uma string. Com o MySQLdb, manipulamos a conexão com o banco de dados MySQL, implementando todos os parâmetros e necessidades para a comunicação e transferência dos dados da planilha.

No processo de codificação da ferramenta, utilizamos a linguagem PHP para gerir a conexão com o banco e apresentar as informações ao usuário. Idealizamos um dos

\footnotetext{
${ }^{1}$ https://www.python.org/
} 
mecanismos de busca por nome do medicamento e ele necessita ser dinâmico para suportar pesquisas após cada letra digitada ou apagada. Com isso foi acoplado ao projeto o framework JQuery, que utilizou da biblioteca já estabelecida JQuery-autocomplete.

Outro mecanismo de busca ocorre através da pesquisa com o código EAN, usado como código de barras no Brasil, aumentando a precisão das buscas. Entretanto, por ser um número com muitos dígitos e parte dos usuários possuírem dificuldades de digitação em dispositivos móveis, acoplamos a entrada desse número com um aplicativo préexistente para leitura de código de barras com a câmera do celular. O usuário decidirá entre preencher o campo digitando o número ou baixar o aplicativo, sendo seu uso de responsabilidade externa ao sistema FarmaJusta.

Para exibir os gráficos estatísticos na interface do administrador, foi utilizado uma biblioteca PHP chamada phplot [AJRepo 2018], ela fornece as funcionalidades de gerar e exibir gráficos como os de barras por exemplo, com o intuito de gerar uma melhor leitura dos dados registrados pelo sistema. Na área da estatística análise de dados, foi acrescentado ao FarmaJusta também o Goolge Analytics ${ }^{2}$, uma ferramenta de análise de clickstream na web[da Cruz 2010]. Ele coleta informações dos usuários e transforma em relatórios, que podem ser usados para avaliar o desempenho de um site ou aplicativo. A interface do sistema Farmajusta foi construída com base nos templates disponibilizados pelo framework Bootstrap [Bootstrap 2018] e da biblioteca JQuery [JQueryFoundation 2018].

\section{Resultados e Discussão}

Conforme citado anteriorimente, para efetivar as buscas, implementamos dois meios de consultas no software são: leitura do código barras ou pela digitação do nome comercial. A busca por código de barras pode ser efetuada por digitação ou utilizando o Barcode Scanner $^{3}$, através da câmera do celular. Com o EAN se reduzem as dúvidas quanto a identificação correta do medicamento. Por exemplo, medicamentos com o mesmo nome porém com dosagens de princípios ativos distintas podem ser fontes de dúvidas para as pessoas. É importante ressaltar essa funcionalidade promove uma experiência mais confortável aos usuários com pouca familiaridade com o universo digital ou mesmo com relação as dosagens.

Caso o usuário escolha digitar o nome do medicamento, ele será auxiliado pelo sistema, já que o mecanismo de busca é equipado com uma ferramenta de sugestão de termos. Quando o medicamento é encontrado via mecanismo de busca por termo, o sistema aconselha o usuário a verificar seu número EAN no código de barras para garantir a identificação correta deste e evitar que o preço seja comparado com o que está sendo fornecido por uma farmácia erroneamente, gerando enganos.

Os usuários do sistema tem como apoio a página Home, onde está a descrição detalhada do sistema. Por exemplo, na página Como Usar, há um tutorial para ensinar como utilizar a aplicação e desfrutar das funcionalidades presentes. Ao acessar a tela Quem Somos é apresentado a equipe desenvolvedora e divulgação do programa PET-SI da UFRRJ, entidade que oferfece o serviço ao público.

Por fim, outro diferencial da aplicação é que todas as buscas efetivadas pelos

\footnotetext{
${ }^{2}$ https://analytics.google.com

${ }^{3}$ https://play.google.com/
} 


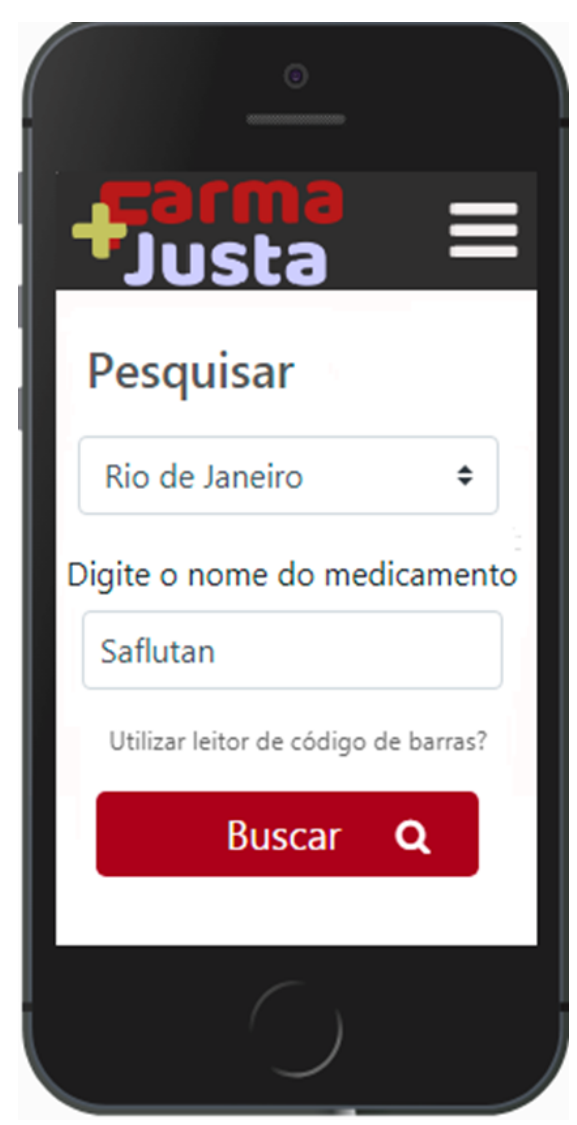

(a) Tela de Pesquisa

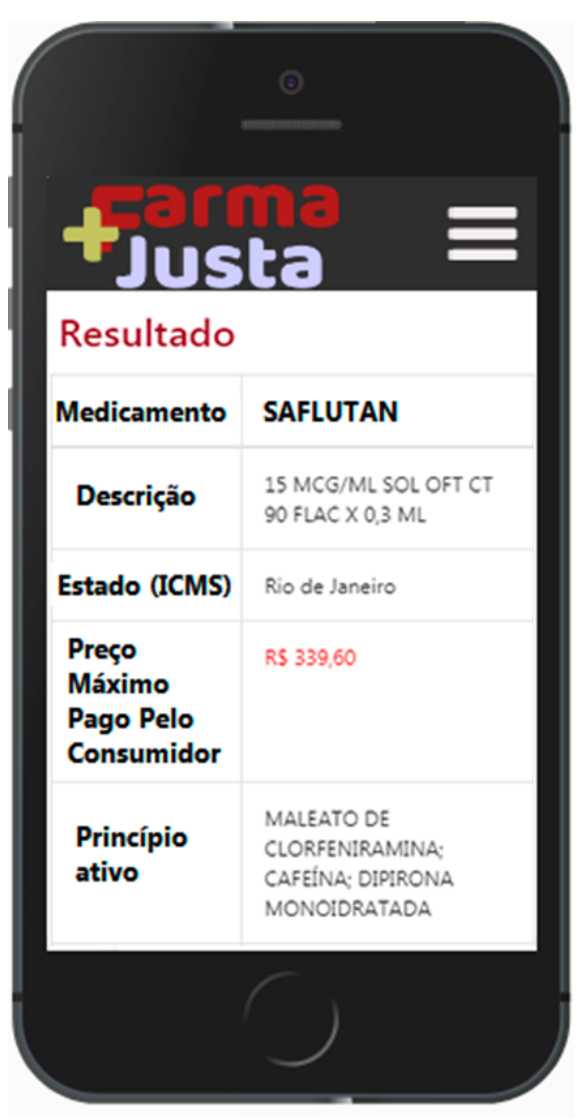

(b) Tela de Resultados

Figura 1. Exemplos de interfaces do sistema FarmaJusta

usuários são armazenadas em uma base de dados, como por exemplo, as informações referentes ao medicamento procurado e ao estado de interesse do usuário. Por exemplo, um indivíduo localizado no estado de Amazonas poderia pesquisar sobre o preço que um medicamento assume no estado de São Paulo apenas por curiosidade, e essa pesquisa seria armazenada no sistema pelo estado de interesse do usuário. No entanto, garantimos a busca da provável localização do usuário com o uso da ferramenta Google Analytics ${ }^{4}$.

Destacamos que cada estado brasileiro possui uma política diferente de preços, onde o preço teto dos medicamentos podem variar de valor de acordo com o ICMS, podemos analisar a figura 2(a). Ela apresenta um exemplo que contém um gráfico da porcentagem de pesquisas realizadas no cenário nacional, e a porcentagem de acessos por cada estado. Diante dessas informações, o Rio de Janeiro foi o estado de maior interesse pelos usuários desde que o sistema foi disponibilizado na rede mundial de computadores, em julho de 2018.

Outras informações obtidas a partir da análise do gráfico de medicamentos mais pesquisados no software Fig.2(b), é que o princípio ativo, por exemplo a levotiroxina Sódica foi um dos mais pesquisados no Farmajusta durante o período de testes. A Levotiroxina Sódica é o componente que compõe o medicamento Puran T4, que atua na terapia de reposição ou suplementação hormonal em pacientes com hipotireoidismo (produção

\footnotetext{
${ }^{4}$ https://analytics.google.com
} 
insuficiente de hormônio pela glândula tireóide) de qualquer causa.

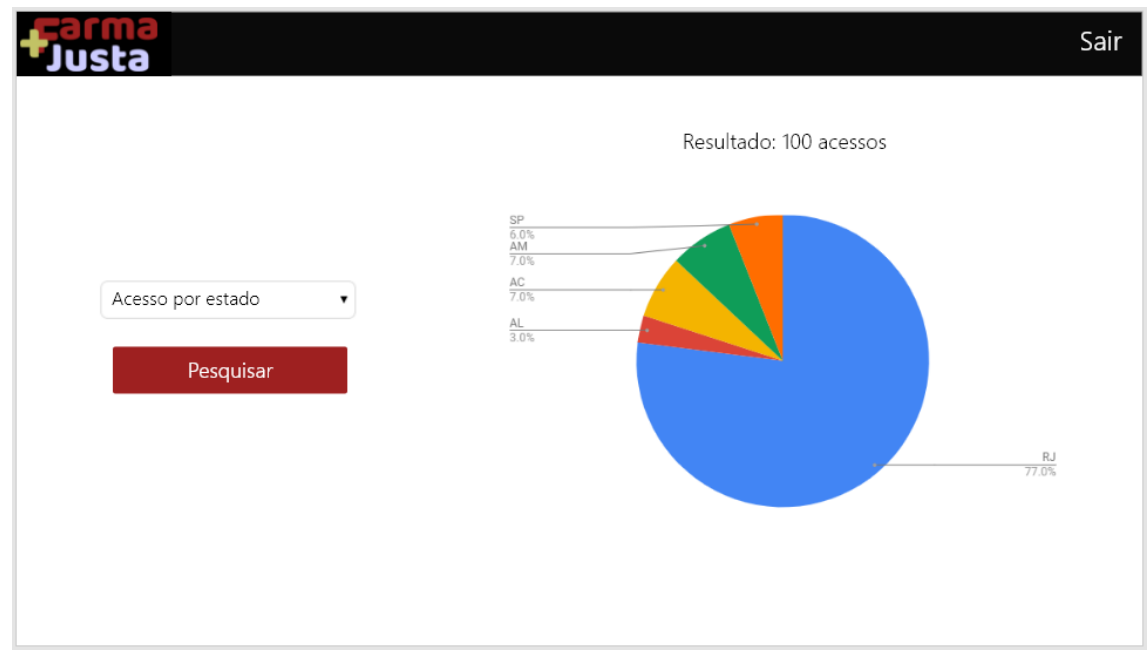

(a) Tela administrativa, que exibe a quantidade de buscas realizadas no sitema.

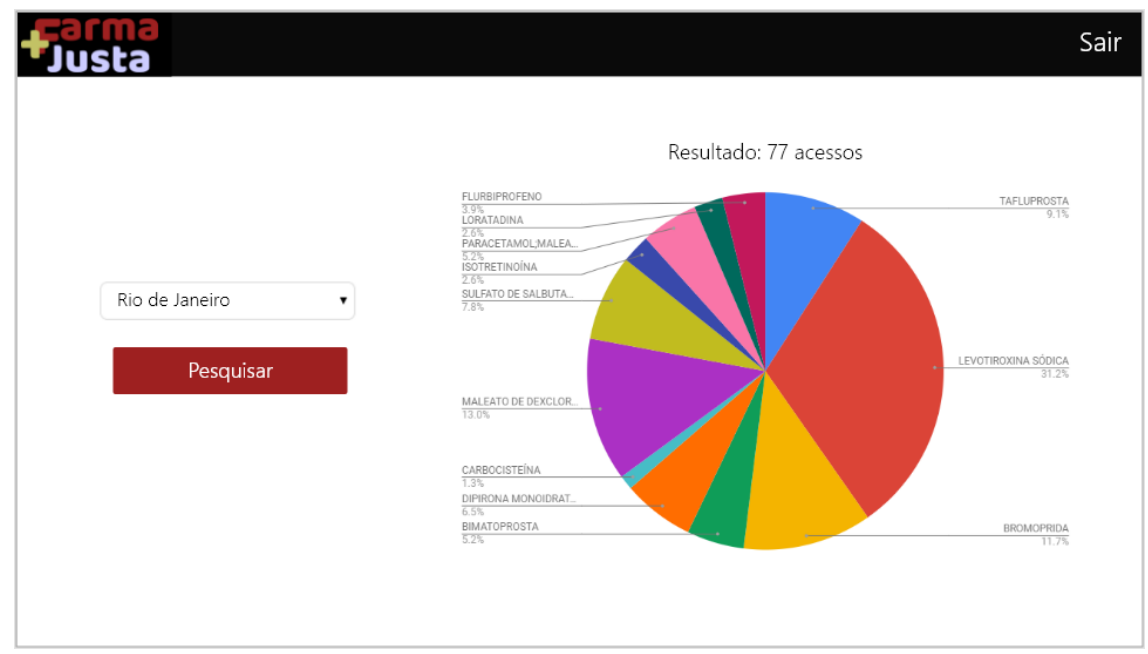

(b) Tela administrativa, que exibe a quantidade de buscas realizadas no sitema.

Figura 2. Interfaces do Sistema FarmaJusta

A versão corrente do sistema foi concebida para armazenar dados de forma anônima sobre as consultas realizadas pelos usuários do FarmaJusta. Assim, se estima que o sistema poderá ser futuramente uma ferramenta auxiliadora no estudos complementares da epidemiologia e do mapeamento da saúde pública do povo brasileiro.

\section{Conclusão}

O FarmaJusta é uma ferramenta aberta e gratuita que foi desenvolvida na tríade ensinopesquisa-extensão. O sistema foi desenvolvido com o propósito de contribuir com a difusão dos dados aberto e conhecimentos sobre saúde pública para a população brasileira. O sistema foi concebido para ser simples e democratizar a acesso aos dados abertos governamentais. Os usuários podem acessá-lo de qualquer tipo de dispositivo sem que percam a legibilidade quando estes possuem tamanho de tela reduzida, tornando o visual atrativo e seu funcionamento intuitivo para facilitar o manejo. 
Também foi atribuído ao sistema a possibilidade de ser uma aplicação que auxilie no estudo da epidemiologia, e que também possa realizar um mapeamento dos medicamentos procurados no Brasil, auxiliando à área da saúde pública. A ferramenta é toralmente gratuita e está disponível em em r1.ufrrj.br/farmajusta. Como trabalhos futuros se pretende, realizar experimentos mais amplos, registrar a ferramenta no Conselho de ética na pesquisa da UFRRJ para reavaliar as interfaces da ferramenta com grupos de pessoas da terceira idade ou portadoras de deficências.

\section{Agradecimentos}

Agradecemos ao MEC/FNDE pelas bolsas e pelo financiamento do programa PET, e ao médico Renato Cerceau ( $\mathrm{PhD}$ ) pelo suporte prestado, trazendo conhecimentos para melhor compreender as necessidades da área da saúde Pública. Agradecemos também a Agência Nacional de Saúde Suplementar (ANS).

\section{Referências}

AJRepo (2018). Phplot is a graph library for dynamic scientific, business, and stockmarket charts and graphs. Online; Acessado em Agosto de 2018.

Bootstrap (2018). Build responsive, mobile-first projects on the web with the world's most popular front-end component library. Online; Acessado em Agosto de 2018.

Brasil (1988). Constituição da república federativa do brasil. Online; Acessado em Agosto de 2018.

Cruz, S. M. S., Quispe, F. E. M., Oliveira, G. S., Leonardo, J. S., Maia, L. F. M. P., Yagui, M. M. M., Chan, V. D., Araújo, Y. L., and RODRIGUES, L. M. O. C. P. (2014). Relato de um experimento piloto de uma fábrica de software baseada em métodos Ágeis.

da Cruz, S. M. S. (2010). Using clickstream and provenance to enhance trust on scientific experiments. Web Science Conference.

da Cruz, S. M. S., Campos, M. L. M., and Mattoso, M. (2009). Towards a taxonomy of provenance in scientific workflow management systems. Congress on Services I.

FOUNDATION, P. S. (2018a). The official home of the python programming language. Online; Acessado em Agosto de 2018.

FOUNDATION, P. S. (2018b). Pypi. pypi - the python package index. Online; Acessado em Agosto de 2018.

GooglePlay (2017). Bulário digital - medicamentos - apps no google play. Online; Acessado em Agosto de 2018.

GooglePlay (2018). Prodoctor medicamentos - apps no google play. Online; Acessado em Agosto de 2018.

Hebert, M. (2018). Preços de remédios podem variar até 300embu e itapecerica.

Hogerzeil HV, M. Z. (2011). The world medicines situation 2011: access to essential medicines as part of the right to health. Online; Acessado em Agosto de 2018.

JQueryFoundation (2018). jquery is a fast, small, and feature-rich javascript library. Online; Acessado em Agosto de 2018. 